\title{
SQLCheck: Automated Detection and Diagnosis of SQL Anti-Patterns
}

\author{
Prashanth Dintyala* \\ vdintyala3@gatech.edu \\ Georgia Institute of Technology
}

\author{
Arpit Narechania* \\ arpitnarechania@gatech.edu \\ Georgia Institute of Technology
}

\author{
Joy Arulraj \\ arulraj@gatech.edu \\ Georgia Institute of Technology
}

\begin{abstract}
The emergence of database-as-a-service platforms has made deploying database applications easier than before. Now, developers can quickly create scalable applications. However, designing performant, maintainable, and accurate applications is challenging. Developers may unknowingly introduce anti-patterns in the application's SQL statements. These anti-patterns are design decisions that are intended to solve a problem, but often lead to other problems by violating fundamental design principles.

In this paper, we present SQLCHECK, a holistic toolchain for automatically finding and fixing anti-patterns in database applications. We introduce techniques for automatically (1) detecting anti-patterns with high precision and recall, (2) ranking the anti-patterns based on their impact on performance, maintainability, and accuracy of applications, and (3) suggesting alternative queries and changes to the database design to fix these anti-patterns. We demonstrate the prevalence of these anti-patterns in a large collection of queries and databases collected from open-source repositories. We introduce an anti-pattern detection algorithm that augments query analysis with data analysis. We present a ranking model for characterizing the impact of frequently occurring anti-patterns. We discuss how SQLCHECK suggests fixes for high-impact anti-patterns using rule-based query refactoring techniques. Our experiments demonstrate that SQLCHECK enables developers to create more performant, maintainable, and accurate applications.
\end{abstract}

\section{CCS Concepts}

- Information systems $\rightarrow$ Database utilities and tools.

\section{Keywords}

Anti-Patterns; Database Applications

\section{ACM Reference Format:}

Prashanth Dintyala, Arpit Narechania, and Joy Arulraj. . SQLCheck: Automated Detection and Diagnosis of SQL Anti-Patterns. In Proceedings of the 2020 ACM SIGMOD International Conference on Management of Data (SIGMOD'20), June 14-19, 2020, Portland, OR, USA. ACM, New York, NY, USA, 18 pages.

\footnotetext{
${ }^{*}$ These authors contributed equally to this work.
}

\section{Introduction}

Two major trends have simplified the design and deployment of data-intensive applications. The first is the spread of data science skills to a larger community of developers [24, 39]. Data scientists combine rich data sources in applications that process large amounts of data in real-time. These applications produce qualitatively better insights in many domains, such as science, governance, and industry [14]. The second trend is the proliferation of database-as-a-service (DBaaS) platforms in the cloud $[13,30]$. Due to economies of scale, these services enable greater access to database management systems (DBMSs) that were previously only in reach for large enterprises. DBaaS platforms obviate the need for in-house database administrators (DBAs) and enable data scientists to quickly deploy widely used applications.

Challenge: Designing database applications is, however, non-trivial since applications may suffer from anti-patterns [27]. An anti-pattern (AP) refers to a design decision that is intended to solve a problem, but that often leads to other problems by violating fundamental design principles. APs in database applications can lead to convoluted logical and physical database designs, thereby affecting the performance, maintainability, and accuracy of the application. The spread of data science skills to a larger community of developers places increased demand for a toolchain that facilitates application design without APs since scientists who are experts in other domains are likely not familiar with these antipatterns [24, 39]. Furthermore, the proliferation of DBaaS platforms obviates the need for in-house DBAs who used to assist application developers with finding and fixing APs.

Sharma et al. have designed a tool, called DBDEO, for automatically detecting APs in database applications [41]. They demonstrate the widespread prevalence of APs in production applications. Although their detection algorithm of DBDEO is effective in uncovering APs, it suffers from three limitations. First, the static analysis algorithm suffers from low precision and recall. Second, it does not rank the APs based on their impact. Third, it does not suggest solutions for fixing them. Thus, a developer would need to manually confirm the APs detected by DBDEO, identify the high-impact APs among them, and fix them. 
In this paper, we investigate how to find, rank, and fix APs in database applications. We present a toolchain, called SQLCHECK, that assists application developers by: (1) detecting APs with high precision and recall, (2) ranking the detected APs based on their impact on performance and maintainability, (3) suggesting fixes for high-impact APs.

The main thrust of our approach is to augment code analysis with data analysis (i.e. examine both queries and data sets of the application) to detect APs with high precision and recall. We study the impact of frequently occurring APs on the performance, maintainability, and accuracy of the application. We then use this information to rank the APs based on their estimated impact. By targeting frequently occurring APs, we take advantage of our ranking model trained on data collected from previous deployments without needing to share sensitive data (e.g. data sets). Lastly, SQLCHECK suggests fixes for high-impact APs using rule-based query refactoring techniques. The advantage of our approach over DBDEO is that it reduces the time that a developer must expend on identifying high-impact APs and fixing them.

In summary, we make the following contributions:

- We illustrate the limitations of the state-of-the-art tools for identifying APs in database applications and motivate the need for an alternate approach with higher precision and recall $(\$ 2)$.

- We introduce an AP detection algorithm that augments query analysis with data analysis (§4).

- We present a ranking model for characterizing the impact of frequently occurring APs on the performance, maintainability, and accuracy of the application (§5).

- We discuss how SQLCHECK suggests fixes for high-impact APs using rule-based query refactoring techniques (\$6).

- We illustrate the efficacy of SQLCHECK in finding, ranking, and fixing APs through an analysis of 1406 opensource database applications, 15 Django applications, 31 Kaggle databases, and a user study (§8).

\section{Motivation \& Background}

We illustrate the need for detecting and diagnosing APs through a case study, then present an overview of the different types of APs and conclude with a discussion on the impact of APs on the application's performance, maintainability, and accuracy.

\subsection{Case Study: GlobaLeaks}

We illustrate the problems introduced by APs through a case study of GlobaLeaks, an open-source application for anonymous-whistleblowing initiatives [17]. The application

\begin{tabular}{rccc} 
Tenant_ID & Zone_ID & Active & User_IDs \\
\hline T1 & Z1 & True & U1 , U2 \\
T2 & Z3 & True & U3 ; U4
\end{tabular}

(a) Tenants Table

\begin{tabular}{rccc} 
User_ID & Name & Role & Email \\
\hline U1 & N1 & R1 & E1 \\
U2 & N2 & R2 & E2 \\
U3 & N3 & R3 & E3 \\
U4 & N4 & R4 & E4 \\
& (b) Users Table &
\end{tabular}

Figure 1: GlobaLeaks Application - List of tables.

supports a multi-tenancy feature to enable multiple organizations to accept submissions and direct them to different endpoints within a single deployment of the application.

EXAMPLE 1: Figure 1 presents the logical database design of the two tables associated with this feature ${ }^{1}$. Since a given tenant can serve multiple users (i.e., one-to-many relationship), the application developer decided to store this information as a comma-separated list of user identifiers in the User_IDs column of Tenants table. While this multi-valued attribute design pattern captures the relationship between the two entities without introducing additional tables or columns, it suffers from performance, maintainability, and data integrity problems. We illustrate these problems using a set of tasks and associated SQL queries executed by the application.

TASK \# 1: The developer is interested in retrieving the tenants that a user is associated with. We cannot use the equality operator in SQL to solve this task since the users are stored in a comma-separated list. Instead, we must employ patternmatching expressions to search for that user:

$/ *$ List the tenants that a user is associated with */ SELECT * FROM Tenants WHERE User_IDs LIKE ' $[[:<:]]$ U1[ [:>:]]';

TASK \#2: Consider the task of retrieving information about the users served by a tenant. This query is also computationally expensive since this involves joining the commaseparated list of users to matching rows in the Users table. Joining two tables using an expression prevents the DBMS from using indexes to accelerate query processing [18]. Instead, it must scan through both tables, generate a cross product, and evaluate the regular expression for every combination of rows.

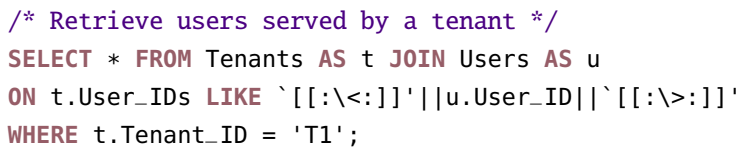

Data Integrity Problems: Another major limitation of this approach is that the developer implicitly assumes that

${ }^{1}$ We distilled the essence of this AP for the sake of presentation. 


\begin{tabular}{rccc} 
User_ID & Name & Role & Email \\
\hline U1 & N1 & R1 & E1
\end{tabular}

(a) Users Table

\begin{tabular}{rcc} 
Tenant_ID & Zone_ID & Active \\
\hline T1 & Z1 & True \\
T2 & Z2 & True
\end{tabular}

(b) Tenants Table

\begin{tabular}{cc} 
Tenant_ID & User_ID \\
\hline T1 & U1 \\
T1 & U2 \\
T2 & U3 \\
T2 & U4 \\
(c) Hosting Table
\end{tabular}

(c) Hosting Table
Figure 2: Refactored GlobaLeaks Application - List of tables.

users will be stored as a list of strings separated by a comma. This implicit assumption might later be violated by a developer entering users separated by another delimiter, such as a semi-colon (e.g. "U6; U7"). This is feasible since the DBMS is not explicitly enforcing that the string should be separated by a particular character. Given this new data, the developer must update all the queries operating on the User_IDs column to handle the usage of multiple separator characters. Furthermore, it is not feasible for the DBMS to enforce a referential integrity constraint between these columns: (1) User_IDs in Tenants, and (2) User_ID in Users. This is because the former column encodes the comma-separated list as a string. So, it is possible for a user in the former column to not have a corresponding tuple in the latter column.

2.1.1 Solution: Intersection TableWe can eliminate this AP by creating an additional intersection table to encode the many-to-many relationship between tenants and users ${ }^{2}$. This table references the Tenant and User tables. In Figure 2, the Hosting table implements this relationship between the two referenced tables.

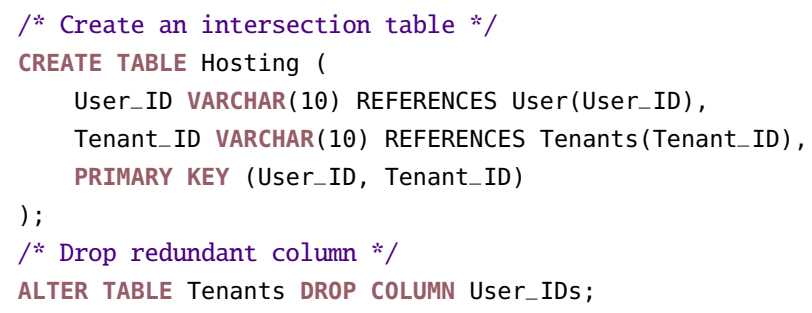

We will next illustrate how this AP-free logical design enables simpler queries for all of the tasks.

TAsks \#1 AND \#2: It is straightforward to join the Tenants and Users tables with the Hosting table to solve the first two tasks. These queries are easy to write for developers and easy to optimize for DBMSs. The DBMS can now use an index on User_IDs to efficiently execute the join instead of matching regular expressions.

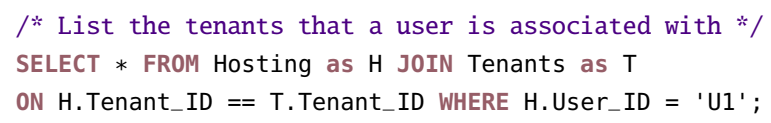

${ }^{2}$ That is, each user may be associated with multiple tenants, and likewise each tenant may serve multiple users.

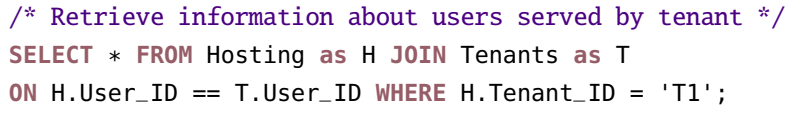

Data Integrity Problems: The developer can delegate the task of ensuring data integrity to the DBMS by specifying the appropriate foreign key constraints. The DBMS will enforce these constraints when data is ingested or updated.

\subsection{Classification of Anti-Patterns}

We compiled a catalog of APs based on several resources that discuss best practices for schema design and query structuring [21, 25, 27, 41]. Table 1 lists the APs that SQLCHECK targets. These APs fall under four categories:

(1) Logical Design APs: This category of APs arises from violating logical design principles that suggest the best way to organize and interconnect data. It includes the multivalued attribute AP covered in §2.1. The adjacency list AP also falls under this category. It refers to references between two attributes within the same table. Such a logical design is used to model hierarchical structures (e.g. employee-manager relationship). With this representation, however, it is not trivial to handle common tasks such as retrieving the employees of a manager up to a certain depth and maintaining the integrity of the relationships when a manager is removed.

(2) Physical Design APs: The next category of APs is associated with efficiently implementing the logical design using the features of a DBMS. This includes rounding errors and enumerated types APs. The rounding errors AP arises when a scientist uses a type with finite precision, such as FLOAT to store fractional data. This may introduce accuracy problems in queries that calculate aggregates. The enumerated types AP occurs when a scientist restricts a column's values by specifying the fixed set of values it can take while defining the table. However, this AP makes it challenging to add, remove, or modify permitted values later and reduces the application's portability ${ }^{3}$.

3 QUERY APs: Query APs arise from violating practices that suggest the best way to retrieve and manipulate data using SQL. This includes NULL usage and column wildcard usage APs. Developers are often caught off-guard by the behavior of NULL in SQL. Unlike in most programming languages, SQL treats NULL as a special value, different from zero, false, or an empty string. This results in counter-intuitive query results and introduces accuracy problems. The latter AP arises when a developer uses wildcards (SELECT *) to retrieve all the columns in a table with less typing. This AP, however breaks applications on refactoring.

4 DAta APs: Data APs are a subset of APs that SQLCHECK detects by analysing the data (as opposed to queries). This

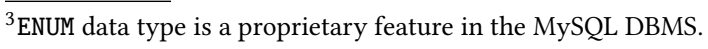




\begin{tabular}{|c|c|c|c|c|c|c|c|}
\hline Category & Anti-Pattern Name & Description & $\mathbf{P}$ & $\mathbf{M}$ & DA & DI & A \\
\hline \multirow{7}{*}{ Logical Design APs } & Multi-Valued Attribute & Storing list of values in a delimiter-separated list violating 1-NF. & $\checkmark$ & $\checkmark$ & $\checkmark^{(\downarrow)}$ & $\checkmark$ & $\checkmark$ \\
\hline & No Primary Key & Lack of data integrity constraints. & $\checkmark$ & $\checkmark$ & $\checkmark^{(\uparrow)}$ & $\checkmark$ & - \\
\hline & No Foreign Key & Lack of referential integrity constraints. & $\checkmark$ & $\checkmark$ & - & $\checkmark$ & - \\
\hline & Generic Primary Key & Creating a generic primary key column (e.g., id) for each table. & - & $\checkmark$ & - & - & - \\
\hline & Data In Metadata & Hard-coding application logic in table's meta-data. & $\checkmark$ & $\checkmark$ & $\checkmark^{(\downarrow)}$ & $\checkmark$ & $\checkmark$ \\
\hline & Adjacency List & Foreign key constraint referring to an attribute in the same table. & $\checkmark$ & - & - & - & - \\
\hline & God Table & Number of attributes defined in the table cross a threshold (e.g., 10) & $\checkmark$ & $\checkmark$ & - & - & - \\
\hline \multirow{6}{*}{ Physical Design APs } & Rounding Errors & Storing fractional data using a type with finite precision (e.g., FLOAT). & - & - & - & - & $\checkmark$ \\
\hline & Enumerated Types & Using enum to constrain the domain of a column. & $\checkmark$ & $\checkmark$ & $\checkmark^{(\downarrow)}$ & - & - \\
\hline & External Data Storage & Storing file paths instead of actual file content in database. & - & $\checkmark$ & - & $\checkmark$ & $\checkmark$ \\
\hline & Index Overuse & Creating too many infrequently-used indexes. & $\checkmark$ & $\checkmark$ & $\checkmark^{(\downarrow)}$ & - & - \\
\hline & Index Underuse & Lack of performance-critical indexes. & $\checkmark$ & $\checkmark$ & $\checkmark^{(\uparrow)}$ & - & - \\
\hline & Clone Table & Multiple tables matching the pattern $<$ TableName>_N & $\checkmark$ & $\checkmark$ & - & $\checkmark$ & $\checkmark$ \\
\hline \multirow{7}{*}{ Query APs } & Column Wildcard Usage & Selecting all attributes from a table using wildcards to reduce typing. & $\checkmark$ & - & - & - & $\checkmark$ \\
\hline & Concatenate Nulls & Concatenating columns that might contain NULL values using || . & - & - & - & - & $\checkmark$ \\
\hline & Ordering by RAND & Using RAND function for random sampling or shuffling. & $\checkmark$ & - & - & - & - \\
\hline & Pattern Matching & Using regular expressions for pattern matching complex strings. & $\checkmark$ & - & - & - & - \\
\hline & Implicit Columns & Not explicitly specifying column names in data modification operations. & - & $\checkmark$ & - & $\checkmark$ & - \\
\hline & DISTINCT and JOIN & Using DISTINCT to remove duplicate values generated by a JOIN. & $\checkmark$ & $\checkmark$ & - & - & - \\
\hline & Too Many Joins & Number of JOINs cross a threshold. & $\checkmark$ & - & - & - & - \\
\hline \multirow{6}{*}{ Data APs } & Missing Timezone & Date-time fields stored without timezone. & - & - & - & - & $\checkmark$ \\
\hline & Incorrect Data Type & Actual data does not conform to expected data type. & $\checkmark$ & - & $\checkmark^{(\downarrow)}$ & - & - \\
\hline & Denormalized Table & Duplication of values. & $\checkmark$ & - & $\checkmark(\downarrow)$ & - & - \\
\hline & Information Duplication & Derived columns (e.g., age from date of birth). & - & $\checkmark$ & - & $\checkmark$ & $\checkmark$ \\
\hline & Redundant Column & Column with NULLS or same value (e.g., en-us) & - & - & $\checkmark^{(\downarrow)}$ & - & - \\
\hline & No Domain Constraint & All values should belong to particular range (e.g., rating) & - & $\checkmark$ & $\checkmark^{(\downarrow)}$ & $\checkmark$ & - \\
\hline
\end{tabular}

Table 1: List of Anti-Patterns: A catalog of APs based on best practices for database application design [21, 25, 27, 41]. They fall under four categories: (1) logical design APs, (2) physical design APs, (3) query APs, and (4) data APs. For each AP we illustrate its impact on five metrics: (1) Performance (P), (2) Maintainability (M), (3) Data Amplification (DA), (4) Data Integrity (DI), and (5) Accuracy (A). $\checkmark$ represents that the given AP affects that metric. $\uparrow$ and $\downarrow$ refer to increase and decrease in data amplification, respectively, when that AP is fixed.

includes the Incorrect Data Type and Information Duplication APs. The former AP arises due to data type mismatches (e.g., storing a numerical field in a TEXT column). This negatively impacts performance and leads to data amplification. The latter AP occurs when a column contains data derived from another column in the same table (e.g., storing age based on date of birth). While this accelerates query processing, it reduces maintainability and leads to data amplification.

\subsection{Impact of Anti-Patterns}

APs in database applications lead to convoluted logical and physical database designs, thereby affecting the performance, maintainability, and accuracy of the application.

1. Performance: An application's performance is often measured in terms of throughput (e.g. the number of requests that can be processed per second) and latency (e.g. the time that it takes for the system to respond to a request) [38]. Optimizing these metrics is important because they determine how quickly an application can process data and how quickly a user can leverage the application to make new decisions.

Consider the tasks presented in $\S 2.3$. We measured the impact of the multi-valued attribute APs on the time taken to

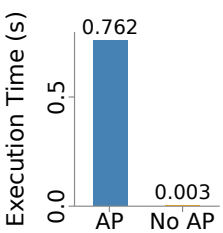

(a) Task \#1

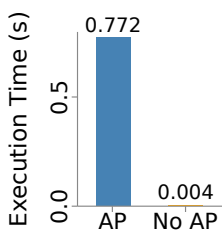

(b) Task \#2

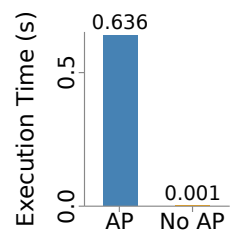

(c) Task \#3
Figure 3: Multi-Valued Attribute AP: Performance impact of the multi-valued attribute AP on the above-mentioned tasks.

execute these tasks. Figure 3 presents the results of this experiment. We defer the discussion of the experimental setup to 8 .2. When we remove this AP, the queries associated with these tasks accelerated by $636 \times, 256 \times$, and $193 \times$ respectively. These results illustrate the importance of fixing APs.

2. Maintainability: The maintainability of an application represents the ease with which the application's design and component queries can be modified to adapt to a changed environment, improve performance or other metrics, or correct faults [20]. Maintainable applications allow developers to quickly and easily add new features, fix bugs in existing features, and increase performance. 


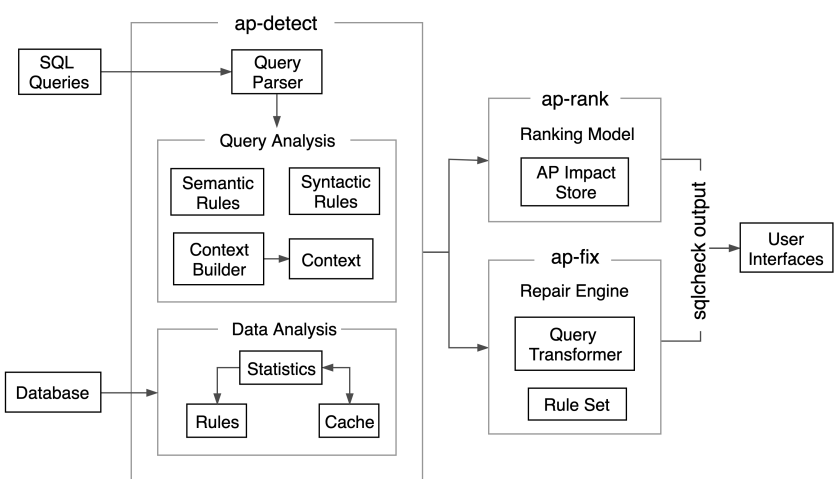

Figure 4: Architecture of sQLCHEck: It takes in a SQL query and database (optional), and produces a ranked list of APs and associated fixes. Internally, SQLCHECK leverages query and data analysis to detect the APs. It then uses a ranking model and query repair engine to generate the desired fixes.

3. ACCURACY: An application's accuracy is measured in terms of the discrepancy between the data stored by a user and that returned by the application. For example, an application that stores fractional numeric data using the FLOAT type in SQL can fail to return certain tuples due to slight discrepancies in their values [27].

Given the impact of APs, we next present an overview of a toolchain that assists developers in eliminating them.

\section{System Overview}

SQLCHECK is geared towards automatically finding, ranking, and fixing APs in database applications. Application developers can leverage SQLCHECK to create more performant, maintainable, and accurate database applications. SQLCHECK contains three components for: (1) detecting APs with high precision and recall, (2) ranking the detected APs based on their impact, and (3) suggesting fixes to application developers for high-impact APs.

Workflow: We envision that application developers will use SQLCHECK in the following manner. A developer will deploy SQLCHECK on their local machine and connect it to the target application (i.e., queries and data sets). (1) The first component, ap-detect, will then perform static analysis of the queries to detect APs. To increase precision and recall, ap-detect will profile the application's data and meta-data (§4). 2 Next, ap-rank will examine the APs detected by ap-detect in the target application and rank them based on their estimated impact (§5). 3 The third tool, ap-fix, will suggest fixes for the high-impact APs identified by ap-rank using rule-based query transformations (§6). (4) Lastly, SQLCHECK will optionally upload the APs detected in the application to an online AP repository with the permission of the developer. As new performance data is collected over time, ap-rank will retrain its ranking model to improve the quality of its decisions.

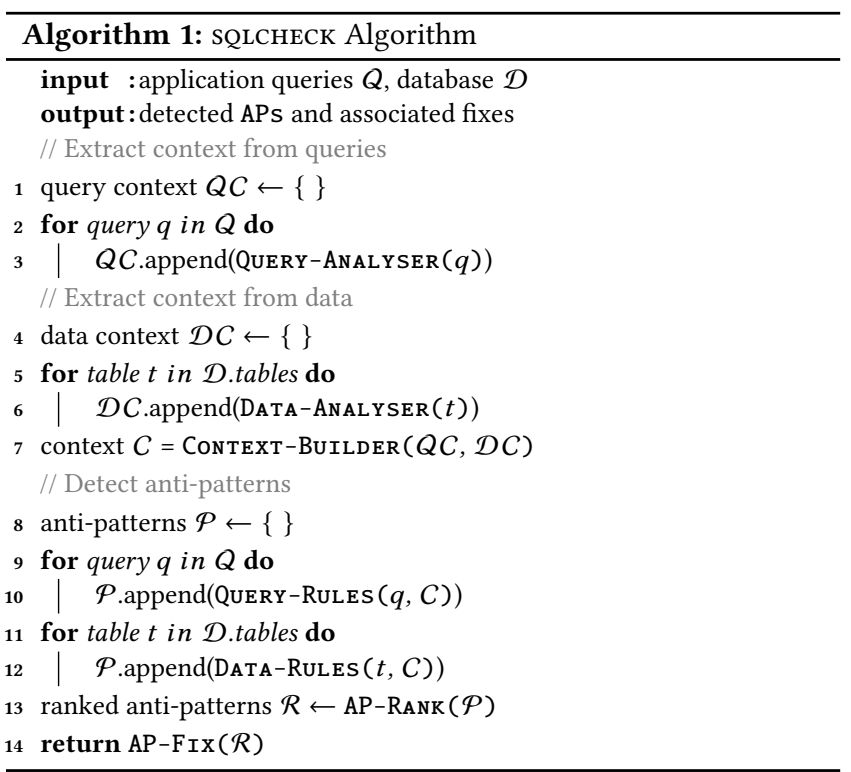

\section{Finding Anti-Patterns}

In this section, we present the techniques used by ap-detect for identifying APs in a given application. We begin with an overview of the SQLCHECK algorithm.

Overview: As shown in Algorithm 1, ap-detect takes a list of SQL queries executed by the application and a connection to the database server as input. It constructs an application context using the given inputs. First, it uses a query analyser to extract context from the queries (e.g., column names, table names, predicates, constraints, and indexes). It tailors the analysis based on the type of SQL statement. We present the query analyser in $\$ 4.1$. Next, it uses a data analyser to extract context from the tables in the database (e.g., data distribution and format of each column). We present the data analyser in $§ 4.2$. Based on the constructed application context, ap-detect uses a set of rules for identifying APs in the given queries ${ }^{4}$. These rules are general-purpose functions that leverage the overall context of the application: (1) queries, (2) data, and (3) meta-data. The APs identified using such query and data analyses are then ordered by ap-rank, which will be covered in $\S 5$.

\subsection{Query Analysis}

ap-detect begins by analysing the SQL queries $Q$ executed by the target database application. It detects APs in the given queries in two phases: (1) intra-query detection and (2) inter-query detection. During the first phase, it identifies APs in each query $q$ in $Q$. During the second phase, it leverages the entire context of the application (i.e., other queries in $Q$

\footnotetext{
${ }^{4}$ This includes both data definition language (DDL) and data manipulation language (DML) commands [11].
} 
and logical design of the database $D$ ) to detect more complex APs. We next discuss these techniques in detail.

(1) Intra-Query Detection: ap-detect applies a set of query rules on the given query $q$. Each query rule consists of a general-purpose function to identify the existence of the target AP in $q$. Rules can range from a simple, patternmatching function that uses a set of regular expressions to complex functions that leverage the inferred context of the application. In order to support diverse SQL dialects, ap-detect leverages a non-validating SQL parser, called sqlparse [2], to process the SQL statement. This parser supports multiple dialects by virtue of its non-validating parsing logic. However, unlike a typical DBMS parser [33], it does not generate a semantically-rich parse tree. We address this limitation by annotating the parse tree returned by sqlparse. ap-detect and ap-fix use this annotated parse tree for finding APs and suggesting fixes, respectively. The tree-structured representation (as opposed to the raw SQL string) allows recursive application of rules and improves the extensibility of the rule system.

ЕхамpLE 2: Consider the following SQL statement for inserting a record into the TENANT table:

INSERT INTO Tenant VALUES ('T1', 'Z1', True, 'U1,U2');

This DML statement fails to correctly function when the schema of TENANT table evolves. For instance, if we drop the User_IDs column and add a new column termed Description, it would incorrectly insert values into the table. This implicit column usage AP also reduces the maintainability of the application. This is because explicitly specifying the column names improves the readability of the query for another developer who is trying to infer the values being inserted into each column. ap-detect identifies this AP by checking whether the column names are present in the INSERT statement. An intra-query detection rule is sufficient to detect the existence of this AP. However, to suggest a fixed INSERT statement, ap-fix needs the application's context (i.e., the schema of the TENANT table).

2 INTER-QUERY DETECTION: The intra-query detection technique suffers from low precision and recall as it does not leverage the relationship between queries in the application which is critical for detecting complex APs.

EXAMPLE 3: Consider the No Foreign Key AP in GlobaLeaks [17]. There are two tables: TENANT and QUESTIONNAIRE. The Tenant_ID column should connect these two tables. However, the DDL statement of the QUESTIONNAIRE table does not define this foreign key relationship. Since the intra-query detection technique applies the rules to each query independently, it is unable to detect this AP by separately examining the two DDL statements. ap-detect can detect the missing

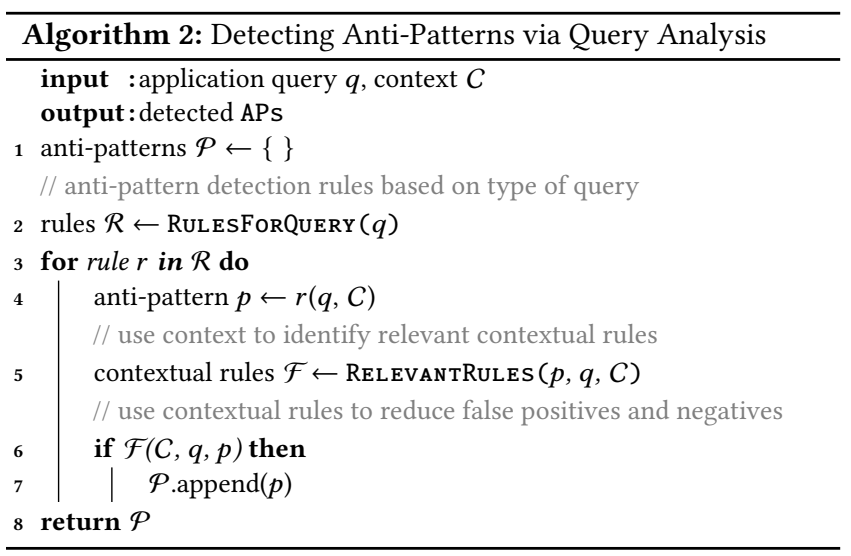

foreign key only if it considers both DDL statements along with the JOIN condition in the SELECT query as follows.

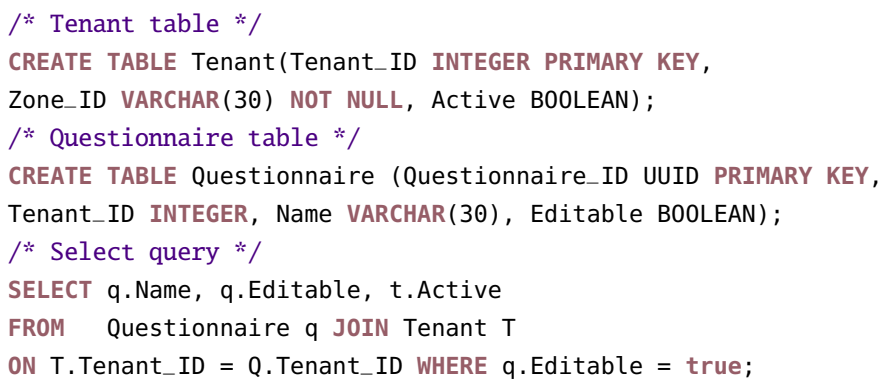

We address the limitations of the intra-query detection technique by constructing the application's context. The context contains two components: (1) the schema and (2) the queries associated with the application. The AP detection rules utilize the context to resolve cases where the presence or absence of an AP cannot be determined with high precision by only looking at a given query.

Algorithm 2 presents the algorithm used for detecting APs by analyzing queries. The ContextBuilder constructs the context using the analysed queries and the database. The context exports a queryable interface for applying contextual rules on the queries, schema, and other application-specific metadata. If the database is not available, the ContextBuilder leverages the DDL statements to construct the context. Given the context, ap-detect first applies a set of AP detection rules based on the type of the query. It then uses the context to identify the relevant context specific rules that are subsequently applied to reduce false positives and negatives.

Limitation: The inter-query detection technique also suffers from false positives and negatives. Consider the multivalued attribute AP discussed in Example 1. ap-detect uses a pattern-matching rule (i.e., regular expression) for detecting this AP in SELECT queries containing string processing tricks. However, this rule can result in: (1) false negatives if the delimiter-separated strings are handled externally in 


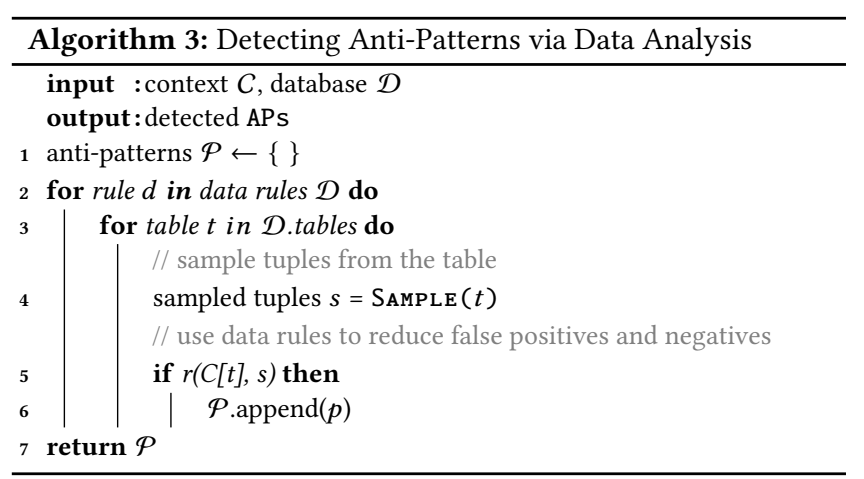

the application code, and (2) false positives if the delimiter is used for an alternate purpose in application (e.g., ADDRESS attribute). Thus, it is not feasible to identify this APs with high precision and recall by only examining the SQL queries. We next discuss how ap-detect extracts and utilizes context from the tables in the database to overcome this limitation.

\subsection{Data Analysis}

ap-detect leverages data analysis to improve the precision and recall of anti-pattern detection. It uses a data analyzer to profile the contents of the database used by the application and uses it to augment the context described in $§ 4.1$. This information is also used for retrieving the relevant contextual rules while detecting APs via query analysis.

For example, in case of the multi-valued attribute AP (Example 1), ap-detect uses a data rule that checks whether a particular column contains delimiter-separated strings. It first checks the data type of the column. If the column is a VARCHAR or TEXT field, it samples the columnar data and checks whether that contains delimiter-separated strings. In case of the TENANT table, as shown in Figure 1a, the User_IDs column contains comma-separated strings. Even if the query rules are unable to detect this AP, the data rule will correctly flag this column as suffering from the MVA AP.

The data analyzer first scans the database to collect: (1) the schemata of the component tables, and (2) the distribution of the data in the component columns (e.g., unique values, mean, median, etc.). It then collects samples from each table in the examined database. ap-detect applies a set of rules for determining the existence of APs in the sampled data. If one of these rules is activated, then ap-detect appends the associated anti-pattern to the list of APs sent to ap-rank.

ExAMPLE 4: Consider the following AP in GlobaLeaks. The Role column in the USER table represents the roles assumed by the users. The developer chose to encode this data as a STRING field with a constraint on the field's domain.

ALTER TABLE User ADD CONSTRAINT User_Role_Check

CHECK (ROLE IN ('R1', 'R2', 'R3'));
We refer to this as the Enumerated Types AP. In this case, the data analyzer extracts the type information of the Role column and notices that it as a STRING field. It then samples the data in the column. ap-detect uses the context to compute the ratio of distinct values to the number of tuples. If this ratio is greater than a given threshold, it detects this AP.

Since data analysis is computationally expensive (e.g., sampling), ap-detect reuses the constructed context across several checks. The data analyzer periodically refreshes the context over time. It also refreshes the context whenever the schema evolves. ap-detect allows the developer to configure the tuple sampling frequency and the thresholds associated with activating data rules.

Rule Complexity: ap-detect supports complex, generalpurpose rules that leverage the overall context of the application. Example 5 illustrates the complexity of rules.

ExAmple 5: The Index Overuse AP is associated with the creation of too many infrequently-used indexes. For instance, consider these three indexes in the TENANT table.

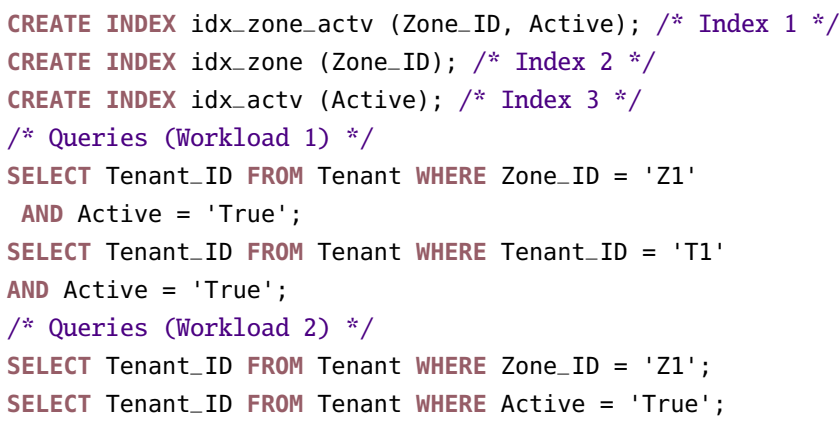

Depending on the workload, ap-detect marks different set of indexes as potentially exhibiting this AP. It leverages the context to determine the list of constructed indexes. For the first workload, it marks the second and third indexes as redundant since these queries will leverage the index on Tenant_ID. For the second workload, it marks the first index as redundant since these queries will leverage the second and third indexes. Thus, ap-detect supports complex rules.

\section{Ranking Anti-Patterns}

In this section, we present the algorithm used by ap-rank for ordering the APs identified by ap-detect. We begin with an overview of the metrics collected by ap-rank for ordering the APs. We then present the model used by ap-rank.

\subsection{Metrics for Ranking Anti-Patterns}

ap-rank collects six metrics for each AP. These metrics are subsequently used by the model for ordering the APs.

(1) Read and Write Performance (RP, WP): This metric characterizes the impact of the AP on the application's performance. We measure the time taken to execute different 


\begin{tabular}{rc} 
Role_ID & Role_Name \\
\hline 1 & R1 \\
2 & R2 \\
3 & R3
\end{tabular}

(a) Role Table

\begin{tabular}{rccc} 
User_ID & Name & Role & Email \\
\hline U1 & N1 & 1 & E1 \\
U2 & N2 & 2 & E2 \\
U3 & N3 & 2 & E3 \\
U4 & N4 & 3 & E4
\end{tabular}

(b) User Table

Figure 5: Refactored GlobaLeaks Application - List of tables.

$$
\begin{gathered}
\mathcal{S}_{r p}(x), \mathcal{S}_{w p}(x), \mathcal{S}_{m}(x)=\min (1, x / 5) \\
\mathcal{S}_{d a}(x)=\min (1, x / 8) \\
\mathcal{S}_{d i}(x), \mathcal{S}_{a}(x)=x / / x \in\{0,1\} \\
\text { score }=\mathcal{W}_{r p}{ }^{*} \mathcal{S}_{r p}(\mathcal{R P})+\mathcal{W}_{w p}{ }^{*} \mathcal{S}_{w p}(\mathcal{W P})+ \\
\mathcal{W}_{m}{ }^{*} \mathcal{S}_{m}(\mathcal{M})+\mathcal{W}_{d a}{ }^{*} \mathcal{S}_{d a}(\mathcal{D} \mathcal{A})+ \\
\mathcal{W}_{d i}{ }^{*} \mathcal{S}_{d i}(\mathcal{D} \mathcal{I})+\mathcal{W}_{A} * \mathcal{S}_{a}(\mathcal{A})
\end{gathered}
$$

Figure 6: Ranking Model - Formulae for measuring the impact of APs.

\begin{tabular}{ccccccc} 
& $\mathcal{W}_{r p}$ & $\mathcal{W}_{w p}$ & $\mathcal{W}_{m}$ & $\mathcal{W}_{d a}$ & $\mathcal{W}_{d i}$ & $\mathcal{W}_{a}$ \\
\hline$C 1$ & 0.7 & 0.15 & 0.05 & 0.04 & 0.02 & 0.02 \\
$C 2$ & 0.4 & 0.4 & 0.1 & 0.04 & 0.02 & 0.02
\end{tabular}

(a) Ranking model configurations

\begin{tabular}{lcccccc} 
& $\mathcal{S}_{r p}$ & $\mathcal{S}_{w p}$ & $\mathcal{S}_{m}$ & $\mathcal{S}_{d a}$ & $\mathcal{S}_{d i}$ & $\mathcal{S}_{a}$ \\
\hline Index Underuse & $1.5 \mathrm{x}$ & 0 & 0 & 0 & 0 & 0 \\
Enumerated Types & 0 & $>10 \mathrm{x}$ & 2 & 1 & 0 & 0 \\
& \multicolumn{2}{c}{ (b) Impact of APs } & & &
\end{tabular}

Figure 7: Ranking Model Configurations - Illustration of the impact of the ranking model configuration on the ordering of APs.

types of frequently-observed queries in the presence and absence of each AP. For this analysis, we focus on the following types of queries: (1) a lookup query that retrieves a set of records from a table based on a highly selective predicate (SELECT), (2) an aggregation query that computes the sum of all the elements in a column (SUM), (3) a join query that combines the records in two tables in an application based on a join predicate (JOIN)), and (4) an update statement that modifies a set of records in a table based on a highly selective predicate (UPDATE). ap-rank uses the results of this quantitative analysis to estimate the potential speedup in executing the target application's queries by fixing an AP. For example, fixing the multi-valued attribute AP (Example 1) accelerates lookup and join queries by $636 \times$ and $256 \times$, respectively.

(2) Maintainability (M): The next metric appraises the impact of each AP on the maintainability of the application. We conduct a qualitative analysis of the number of changes $(C)$ needed in an application to support a new task in the presence and absence of each AP. This determines the degree of refactoring necessitated by the AP. If $C$ is linearly or superlinearly dependent on the number of queries $(Q)$ present in the application, then ap-rank will prioritize this AP. In contrast, a design wherein $C$ is independent of $Q$ improves the extensibility of the application.
Consider the enumerated types AP (Example 4). If the developer would like to rename a particular Role (e.g., R2 $\mapsto R 5$ ), they would need to execute the following queries:

ALTER TABLE User DROP CONSTRAINT IF EXISTS User_Role_Check; UPDATE User SET Role='R5' WHERE Role='R2';

Figure $5 \mathrm{~b}$ illustrates an alternate design wherein only one query (i.e., $C=O(1)$ ) is sufficient for this refactoring.

UPDATE Role SET Role_Name='R5' WHERE Role_Name='R2' ;

3 Data Amplification (DA): The next metric appraises the impact of APs on data amplification. AP-free design can shrink the storage footprint of an application.

Consider the enumerated types AP in GlobaLeaks (Example 4). The Role column can take the following STRING values: (R1, R2, and R3). Repeatedly storing these STRING values increases the storage footprint of the application. The alternate design presented in Figure 5b addresses this limitation by introducing a ROLE table and encoding the Role column in the USER table using INTEGER values: $(1,2$, and 3 ). In addition to reducing data amplification, it allows the developer to utilize foreign key constraints (e.g., to ensure that every user can only take on one of these roles) ${ }^{5}$.

(4) Data Integrity (DI): The third metric characterizes the impact of each AP on data integrity. We examine how an AP affects the integrity of the application. For instance, consider the multi-valued attribute AP in GlobaLeaks (Example 1). If a user with User_ID u1 is deleted from the USER table, we need to manually execute another query to delete the u1 string from the comma-separated User_IDs field.

UPDATE Tenants SET User_IDs = REPLACE(User_IDs, ',u1', '') WHERE User_IDS LIKE '\%U1\%';

If this query is not executed, the data integrity constraint will be violated. In contrast, if the database contains an intersection table as shown in Figure 2c, we can leverage DBMS features for preserving integrity constraints (e.g., cascaded deletes as shown in §2.1).

(5 Accuracy (A): This metric characterizes the impact of the AP on the accuracy of the returned results. Consider the no foreign key AP (Example 3). In the QUESTIONNAIRE table, since there is no foreign key linking the Tenant_ID columns in the Tenant and QUESTIONNAIRE tables, delete operations will not be cascaded. The resultant dangling references lead to tuples with NULL values when these tables are joined.

\subsection{Model for Ranking Anti-Patterns}

We now present the ranking model that ap-rank uses for ordering the APs identified by ap-detect. Our goal is to prioritize the attention of developers on high-impact APs. The model leverages the metrics presented in §5.1.

\footnotetext{
${ }^{5}$ This relationship can also be preserved using a CHECK constraint [34]. However, this feature reduces performance and maintainability (\$8.2).
} 
ap-rank sorts the APs in the application in decreasing order of their estimated impact on performance, maintainability, and accuracy. To do this estimation, it maps the queries in the application to the standard types of queries that have already been evaluated. It then generates a query-aware ranking of APs in the application. The developer can tailor the weights used by the model for these different features: performance, maintainability, and accuracy. It then sends the ordered list of APs to ap-fix. For APs with multiple candidate fixes, ap-fix suggests the best fix based on the collection of queries present in the application. We defer a discussion of ap-fix to $\S 6$. As new performance data is collected over time, we update the ranking model to improve the quality of its decisions.

Model Components: The model consists of two components: (1) intra-query and (2) inter-query ranking components. The intra-query component ranks the APs detected in each query. It first computes the following metrics for each AP: (1) read performance $(\mathcal{R P}),(2)$ write performance $(\mathcal{W P}),(3)$ maintainability $(\mathcal{M}),(4)$ data amplification $(\mathcal{D} \mathcal{A})$, (5) data integrity $(\mathcal{D} \mathcal{I})$, and (6) accuracy $(\mathcal{A})$.

It then aggregates these metrics using the weights shown in Figure 6. The developer can configure these weights to best meet their applications requirements. For instance, if an application requires higher read performance, the developer can increase the read performance weight. ap-rank uses the computed aggregate score for ranking the APs within a query and to compute the score for each AP.

The inter-query component sorts APs based on their impact on all the queries in the application. The developer can choose one of two inter-query ranking models: 1 based on number of APs in each query (i.e., queries with more APs are ranked higher), or 2 based on the computed score.

Ехамрце 6: Consider a query suffering from the index underuse and enumerated types APs. Figure 7a illustrates two different configurations of the ranking model ( $C 1$ and $C 2$ ). Figure $7 \mathrm{~b}$ lists the metrics associated with the detected APs. The first configuration (C1) prioritises read performance ((e.g., analytical workloads). So, it ranks the index underuse AP (score $=0.21$ ) higher than the enumerated types AP (score $=0.175)$. In contrast, the second configuration $(C 2)$ gives equal priority to both read and write performance (e.g., hybrid transactional/analytical workloads). So, it ranks the enumerated types AP (score $=0.47$ ) higher than the index underuse types AP (score $=0.12$ ). In this manner, ap-rank allows the developer to prioritise APs.

Conflicting Fixes: Fixes for APs detected in an application may conflict with each other. ap-rank assists the developer in resolving these conflicts by prioritising the APs. For instance, consider an application with these two APs:
Too Many Joins and Enumerated Types. To resolve the latter AP, the developer must create a new table for the attribute with an enumerated type (e.g., ROLE table). However, this fix would increase the performance impact of former AP as it would require the developer to connect the newly added table with an additional JOIN in SELECT queries. SQLCHECK orders the detected APs based on the user-specified ranking model. So, if the developer is prioritising read performance, then they may fix the former AP first and ignore the latter one. In this manner, they can iteratively fix the APs in the application based on their impact score from ap-rank.

\section{Fixing Anti-Patterns}

Merely identifying the high-impact APs will not be sufficient since application developers who are experts in other domains are likely not familiar with anti-patterns [24, 39]. ap-fix addresses this problem by automatically suggesting alternate database designs and queries that are tailored to the application. We begin with an overview of the algorithm used by ap-fix. We then describe the query repair engine that ap-fix leverages for rewriting SQL queries in $§ 6.1$.

OVERVIEw: As shown in Algorithm 4, ap-fix takes the following inputs: (1) a list of detected APs, (2) the parse trees of the queries containing those APs, and (3) the context of the application. Depending on the types of APs, it fetches the associated rules for fixing them. Besides targeting the queries containing the APs, ap-fix retrieves the list of queries $\mathcal{I}$ that are also impacted by the AP fix from the application's context. It appends these impacted queries to the list of queries containing APs to construct the list of queries that must be transformed $(\mathcal{Z})$. ap-fix then passes this list to the query repair engine (Line 7). The rule engine checks whether it can generate non-ambiguous query transformations for a given query based on the APs that it contains. If that is the case, then it applies those transformations on the query's parse tree (Line 9). It then transforms the parse tree to a SQL string based on the dialect used by the application. If it cannot generate non-ambiguous transformations, then it returns a textual fix that is tailored based on the context (Line 12). The application developer must subsequently follow the guidance provided in the textual fix to manually resolve the detected APs.

\subsection{Query Repair Engine}

The query repair engine transforms a given SQL statement based on a set of rules for fixing APs. The rule system is instrumental in facilitating our experimentation with statement transformations for two reasons. First, the rule system paradigm makes it easy for ap-fix to exploit the complicated triggering interactions between the repair rules, thereby obviating the need for explicitly laying out the flow of control between rules. Second, the rule system is extensible. This 


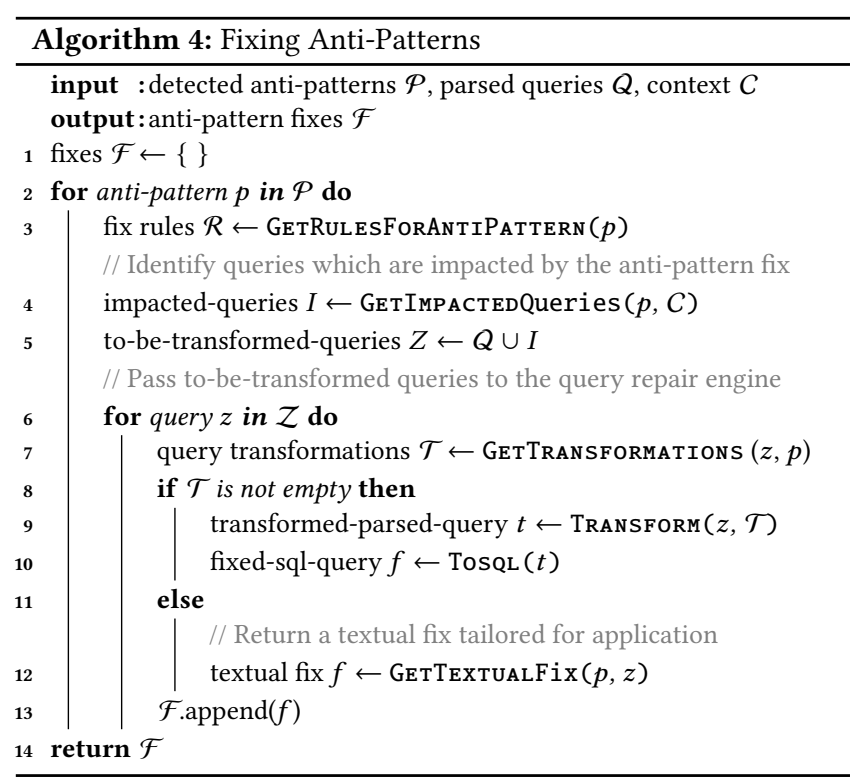

extensibility allowed us to formulate and evaluate tens of transformations over time.

In addition to rewriting existing SQL statements, ap-fix also needs to construct new statements for certain APs. For example, in the case of the multi-valued attribute AP (1), ap-fix first constructs a new HOSTING table and then updates the schema of the TENANTS table, as shown below:

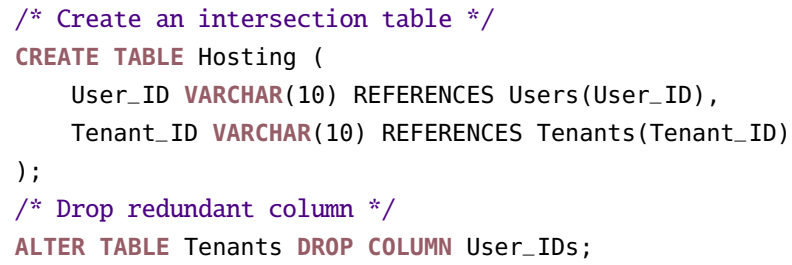

A key challenge for the query repair engine is that it must identify all the queries which are impacted by the antipattern fix and transform them as well. For instance, with the intersection table, ap-fix rewrites the query for retrieving information about the users served by tenant thus:

$/ *$ Retrieve information about users served by tenant */ SELECT * FROM Hosting as $\mathrm{H}$ JOIN Tenants as $\mathrm{T}$

ON H.User_ID $==T$.User_ID WHERE H.Tenant_ID $=$ ' $T 1$ ' ;

Rule Representation: We represent rules in our engine as pairs of functions in a procedural language. Each rule consists of: (1) a detection function (\$4), which does an arbitrary check and sets a flag TRUE or FALSE, and (2) an action function, which, if the condition function sets the flag TRUE, is invoked to take an arbitrary action, such as transforming existing SQL statements and creating new SQL statements.

\section{Implementation}

SQLCHECK is implemented in Python [35] and exports the following three interfaces. (1) Interactive Shell, (2) REST, and (3) GUI. Application developers and SQL IDE developers can leverage these interfaces to either directly interact with SQLCHECK or to integrate it with their own IDEs. We describe these interfaces below:

- Interactive Shell: An SQL application developer can import the SQLCHECK package from a package repository (e.g., PyPI [36]) and directly use the interactive shell interface to execute SQL queries or leverage these sub-modules in other tools.

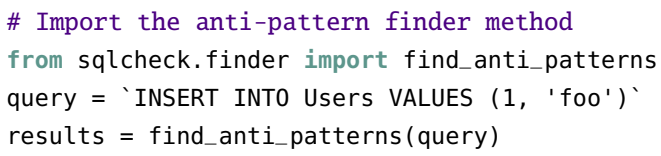

- REST Interface: This interface allows developers to leverage SQLCHECK in applications developed in other programming languages by using web requests via HTTP. We implement this using the Flask web framework [32]. HTTP POST /api/check Body: \{"query":"INSERT INTO Users VALUES (1,'foo')"\}

- GUI Interface: Lastly, this interface is geared towards a wider range of users who are not familiar with application programming. This interface enables users to easily get feedback on their queries by copying them into the input field and is developed using ReactJS [22].

EXTENSIBILITY: SQLCHECK is extensible by design. A developer may add a new AP rule that implements the generic rule interface (name, type, detection rule, ranking metrics, and repair rule) and register it in the SQLCHECK rule registry. A developer may also extend the context builder to augment the application's context for supporting complex rules. Lastly, a developer may replace the non-validating parser with a DBMS-specific parser to increase the utility of the parse tree.

\section{Evaluation}

We evaluated SQLCHECK on a variety of real-world SQL queries to quantify its efficacy in detecting, ranking and fixing APs. We illustrate that:

- Detection: SQLCHECK detects a wider range of APs (26) in real-world DBMS applications compared to DBDEO (11). ap-detect has $48 \%$ fewer false positives and $20 \%$ fewer false negatives than DBDEO resulting in higher precision and recall. SQLCHECK found 32 major APs in 15 real-world web applications.

- Ranking: ap-rank allows the developer to order APs based on their impact. Its ranking model is derived through an empirical analysis of Globaleaks. We show 


\begin{tabular}{r|ccc|cccc} 
AP Name & S & D & Both & TP-S & FP-S & TP-D & FP-D \\
\hline Pattern Matching & 1037 & 524 & 28 & 705 & 332 & 0 & 524 \\
God Table & 27 & 170 & 3344 & 27 & 0 & 0 & 170 \\
Enumerated Types & 414 & 42 & 48 & 411 & 3 & 0 & 43 \\
Rounding Errors & 352 & 7 & 1074 & 329 & 23 & 0 & 7 \\
Data in Metadata & 18 & 584 & 1226 & 18 & 0 & 93 & 491 \\
Adjacency List & 0 & 10 & 93 & 0 & 0 & 0 & 10 \\
\hline Total: & 1848 & 1337 & 5813 & 1588 & 358 & 93 & 3783
\end{tabular}

Table 2: Detection of Anti-Patterns: Comparison of the number of APs identified by SQLCHECK and DBDEO in the query benchmark. Columns $\mathbf{S}$ and $\mathbf{D}$ report the APs detected by only that tool. Column Both lists the APs detected by both tools. TP and FP refer to true and false positives, respectively.

that the average and maximal impact of APs on runtime performance is $4 \times$ and $>10000 \times$, respectively.

- Fixing: We conduct a user study to validate the efficacy of SQLCHECK in fixing APS. SQLCHECK's efficacy in resolving the APs in the queries written by users is $51 \%$. Overall, the participants of the study confirmed that SQLCHECK helped eliminate APs in their applications.

\subsection{Detection of Anti-Patterns}

To our knowledge, DBDEO is the closest system to SQLCHECK. DBDEO is effective in uncovering APs in real-world SQL queries. However, DBDEO's query analysis algorithm suffers from low precision and recall. Furthermore, it differs from SQLCHECK in that it neither ranks the APs based on their impact nor suggest fixes for the detected APs. In this experiment, we compare the AP-coverage and accuracy of SQLCHECK against that of DBDEO.

QUERY BENCHMARK: We download 1406 open-source repositories containing SQL statements from GitHub [23]. We extract around 174 thousand string-quoted embedded SQL statements from these repositories. We then use regular expressions to extract SQL statements from the files contained in these repositories. We evaluate SQLCHECK under two different configurations: (1) with only intra-query analysis, and (2) with both intra- and inter-query analyses. The open-source applications hosted on GitHub only contain SQL queries and not their associated databases. So, SQLCHECK cannot leverage its data analysis techniques in this experiment.

Results: We group and aggregate the detected APs based on their type. The results (details in Table 3) are as follows:

- DBDEO detects 14764 APs (11 types of APs).

- SQLCHECK (only intra-query analysis) detects 86656 APs (18 types of APs).

- SQLCHECK (intra- and inter-query analysis) detects 63058 APs (21 types of APs).

- Coverage and Accuracy: Under both configurations, SQLCHECK detects a wider range of APs compared to DBDEO. With only intra-query analysis enabled, SQLCHECK finds $2.6 \times$ more APs than DBDEO. We attribute this increase in recall to two factors. First, SQLCHECK supports 26 types of APs (DBDEO only supports 11 types.) Second, SQLCHECK uses detection rules that are capable of uncovering different variants of the same AP (e.g., set of regular expressions for identifying Multi-Valued Attribute: (id $\backslash \backslash s+$ regexp) $\mid(i d \backslash \backslash s+l i k e)$ ). This increase in recall also results in higher false positives (i.e., lower precision). Enabling both intra- and inter-query analyses mitigates this problem. Under this configuration, SQLCHECK reports three additional types of AP but $1.8 \times$ fewer APs compared to the prior configuration. This is because it eliminates false positives by leveraging the inter-query context.

- Dialect-Coverage: Qualitatively, both SQLCHECK and DBDEO support a wide range of SQL dialects. We attribute this to their usage of sqlparse, a non-validating parser, that supports diverse dialects. Furthermore, SQLCHECK leverages sqlalchemy to construct the query and context objects in a DBMS-agnostic manner [42].

We next conduct a manual analysis of the APs reported by DBDEO and SQLCHECK in the query benchmark for a subset of APs. We do not examine certain AP (e.g., No Primary Key, Index Underuse) because DBDEO does not report the query in which the AP was detected. The results are shown in Table 2. SQLCHECK has $48 \%$ fewer false positives and $20 \%$ fewer false negatives compared to DBDEO. This illustrates the impact of intra- and inter-query analyses in increasing the precision and recall of AP-detection.

\subsection{Ranking and Repair of Antipatterns}

We next examine the impact of APs on runtime performance. In particular, we compare the query execution time before and after fixing a given AP in a real-world application.

EXPERIment Setup: We aggregate the APs detected in the query benchmark presented in $\$ 8.1$ based on their associated application. We rank these applications based on the frequency and types of detected APs. Based on this ranking, we select the Globaleaks application for this experiment [17].

Globaleaks leverages sqlalchemy (an object-relational mapping (ORM) framework) [42]. We first transform the ORM operations to SQL queries. We then recreate the database schema on a DBMS instance (PostgreSQL v11.2) and load a synthetic dataset containing $10 \mathrm{M}$ records (19 GB across 11 tables). Globaleaks inherently contains ten types of APs. We infuse three additional APs for quantifying their performance impact. For instance, we add comma-separated strings in a column to infuse the multi-valued attribute AP.

We quantify the performance impact of every AP. For each AP, we execute different types of queries in Globaleaks under two configurations: (1) before the AP is fixed, and (2) after the 
AP is fixed using the feedback provided by ap-fix. For each query, we report the average execution time of five runs.

INDEX OVERUSE AP: This AP is associated with the creation of too many infrequently-used indexes. As shown in Figure 8a, the performance impact of this AP is significant for the UPDATE statement. We attribute this to the overhead of maintaining the indices. The update operation is $10 \times$ slower when there are five indices on the field being updated. Thus, it is advisable to only create those indexes whose impact on query processing is significant. Another fix for this AP is to maintain a multi-column index as opposed to maintaining multiple single-column indices.

INDEX UNDE RUSE AP: Figure 8b illustrates the impact of not having important indices on columns. We execute a query which performs a post-grouping aggregation operation. The query execution time drops by $1.3 \times$ when we create an index on the column contained in the GROUP BY clause. This is because the index eliminates the overhead of the grouping operation. Figure 8c illustrates a scenario where fixing this AP reduces performance. We consider a scan query with a predicate on a column with low cardinality. The query executes $3 \times$ slower when it uses the index as opposed to a table scan. We attribute this to the low cardinality of the indexed column [12]. The query analysis rule incorrectly flags this query due to missing indices. SQLCHECK eliminates this false positive by leveraging its data analysis rule which takes cardinality into consideration.

No Foreign Key Exists AP: Figures 8d to 8f illustrate the performance impact of this APs on an UPDATE statement and a scan query. The performance impact is not prominent in both cases since the PostgreSQL does not automatically create an index to maintain the foreign key constraint. An index explicitly constructed by the user accelerates the UPDATE operation by $142 \times$. This AP also has a significant impact on maintainability and data consistency. This is because the foreign key constraint must be preserved by the developer using complex application-level logic. For instance, in case of a cascaded DELETE operation, a developer will need to issue two SQL statements to update the values in both the reference and referencing tables. Otherwise, the referential integrity constraint will not be preserved. This increases the complexity associated with maintaining the application.

ENumerated Types AP: With this AP, the developer uses the CHECK constraint feature of the DBMS [34]. We measure the time taken to update a value of the Role column covered by the constraint ( $\mathrm{R} 2 \mapsto \mathrm{R} 5$ ). This consists of three steps: (1) an ALTER operation to drop the CHECK constraint on the Role column, (2) updating the value using an UPDATE statement based on a predicate, and (3) an ALTER operation to add the constraint back onto the column. In contrast, if the database contains an intersection table as shown in Figure 2c, the same task can be accomplished using an UPDATE statement. Figures $8 \mathrm{~g}$ to $8 \mathrm{i}$ illustrates the performance impact of this AP. Eliminating this AP improves performance by more than $1000 \times$ in case of UPDATE and INSERT operations. The impact is less prominent in case of the scan query due to the overhead of the JOIN operator. The AP-free design improves maintainability by reducing the number of queries required for performing a given task (e.g., Role update). Furthermore, it reduces the storage footprint by reducing data duplication.

SEVERITY OF APs: Impact of an AP depends on the application context (e.g., Enumerated Types AP will not affect performance if the attribute's domain does not change). Certain APs may stem from application requirements. For instance, it may not be possible to simplify a query with Too Many Joins. Lastly, a subset of APs will always have negative impact (e.g., No Foreign Key will always affect data integrity).

\subsection{User Study}

In this experiment, we evaluate the efficacy of SQLCHECK through a user study. We recruited 23 graduate and undergraduate students majoring in Computer Science with varying degrees of expertise in SQL.

Experiment Setup: We tasked the participants to construct a set of SQL queries for a bike e-commerce application. The requirements are twofold: (1) design a performant and extensible database design for this application, and (2) formulate performant SQL queries to support application features. We curated a set of sixteen features that are associated with one or more APs (e.g., shopping cart, list of products).

The participants use the GUI Interface presented in $§ 7$. We track: (1) the original SQL queries developed by the user, (2) the fixes suggested by SQLCHECK for the APs detected in the original queries, and (3) the re-formulated SQL queries developed by the user that incorporate these fixes. We also collect qualitative feedback from the participants about the accuracy and utility of the detected APs and their fixes.

RESULTs: The participants constructed 987 SQL statements. SQLCHECK detected and suggested fixes for 207 APs. Most of the participants ( 20 out of 23 ) took the 187 APs detected in their queries into consideration. They refactored the queries to resolve 96 APs. They ignored the remaining 91 fixes. The reasons for this are twofold: (1) ambiguous fixes (31 fixes), and (2) incorrect fixes (60 fixes) given the requirements of the application. Thus, the participants leveraged $51 \%$ of the fixes suggested by SQLCHECK. If we also include the APs labeled as ambiguous from the participants' perspective, then the efficacy increases to $67 \%$.

We compare the distribution of APs detected in the participants' queries using DBDEO and SQLCHECK. The results are shown in Table 3. There is significant variation in the 


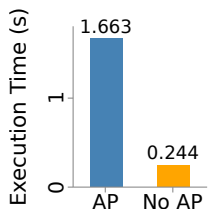

(a) Index Overuse: Update

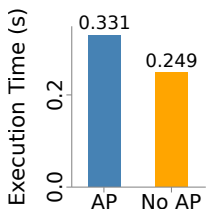

(b) Index Underuse: Grouped Aggregate

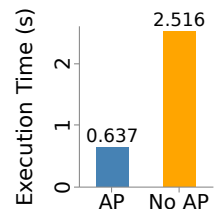

(c) Index Underuse: Scan with Predicate

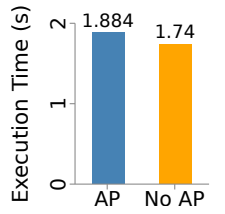

(d) Foreign Key Exists:

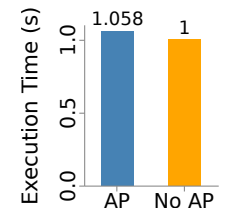

(e) Foreign Key Exists: Select

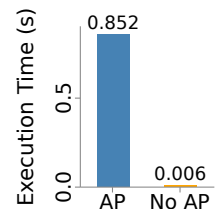

(f) Foreign Key Exists: Update with Index

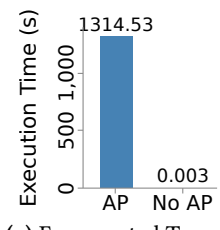

(g) Enumerated Types:

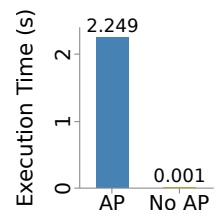

(h) Enumerated Types:

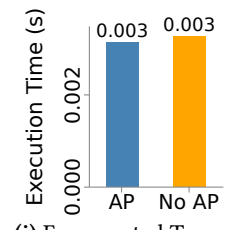

(i) Enumerated Types:

Figure 8: Ranking and Repair of AP: Performance impact of AP on different types of SQL statements.

frequency of APs. For instance, the No Primary Key AP is $14 \times$ more prevalent than the Pattern Matching AP.

We next examine the variance in SQL skills of the participants. The number of queries executed, detected APs, and accepted APs follow these distributions: $(\mu=42.5, \mathrm{Q} 2=46)$, $(\mu=9.35, \mathrm{Q} 2=8)$, and $(\mu=4.8, \mathrm{Q} 2=46)$. The high variance in SQL skills illustrates the need for an automated toolchain for detecting APs and suggesting fixes. The qualitative feedback from the participants indicate that they predominantly found SQLCHECK to be helpful in understanding APs.

\subsection{Web Applications \& Databases}

In this experiment, we first evaluate the efficacy of SQLCHECK in finding, ranking, and suggesting fixes for APs in realworld web applications on GitHub. We apply sQLCHECK on 15 actively-developed Django-based applications [1].

Experiment Setup: We first manually deploy each of these applications on PostgreSQL. We then collect the SQL queries either by running the integration tests or by manually interacting with the application. Lastly, we report the high-impact APs to the developers by either raising issues on GitHub or through the official developer forum. Before reporting the APs, we manually analyse them to study their significance based on the application-specific context. We order the APs impact metrics thus: read performance, maintainability, write performance, accuracy, and amplification.

Results: As shown in Table 4, sQLCHEcK detected 123 APs across these applications (Ref. Section B). We reported 32 APs based on their impact score and the application-specific context. We do not report low severity APs (e.g., Generic Primary Key) and those that require a deeper understanding of application requirements (e.g., Too Many Joins).

We have received responses from all but three of these development teams. Eleven teams acknowledged the existence of APs in their applications. They attribute these APs

\begin{tabular}{r|cc|cc|cc} 
& \multicolumn{2}{|c|}{ GitHub Rep } & \multicolumn{2}{c}{ User Study } & \multicolumn{2}{c}{ Kaggle } \\
Anti-Pattern & D & S & D & S & D & S \\
\hline No Primary Key & 628 & 6875 & 22 & 70 & - & 68 \\
Column Wildcard Usage & 0 & 12313 & 0 & 54 & - & - \\
Data in Metadata & 1907 & 1352 & 43 & 39 & - & 9 \\
Enumerated Types & 90 & 462 & 11 & 30 & - & - \\
Index Underuse & 82 & 506 & 40 & 30 & - & - \\
God Table & 3514 & 3371 & 22 & 28 & - & - \\
Implicit Columns & 0 & 26488 & 0 & 24 & - & - \\
Readable Password & 0 & 295 & 0 & 20 & - & - \\
Clone Table & 1990 & 516 & 21 & 12 & - & - \\
Rounding Errors & 1081 & 1426 & 91 & 10 & - & - \\
Generic Primary Key & 0 & 5123 & 0 & 8 & - & 25 \\
Multi-Valued Attribute & 2539 & 1503 & 3 & 6 & - & 20 \\
Pattern Matching & 552 & 1065 & 25 & 5 & - & - \\
Adjacency List & 103 & 93 & 0 & 0 & - & - \\
No Foreign Key & 0 & 1389 & 0 & 0 & - & 10 \\
External Data Storage & 0 & 63 & 0 & 0 & - & - \\
Index Overuse & 228 & 228 & 0 & 0 & - & - \\
Concatenate Nulls & 0 & 63 & 0 & 0 & - & - \\
Ordering by Rand & 0 & 27 & 0 & 0 & - & - \\
Distinct and Join & 0 & 4 & 0 & 0 & - & - \\
Too many Joins & 0 & 4 & 0 & 0 & - & - \\
Missing Timezone & - & - & - & - & - & 12 \\
Incorrect Data Type & - & - & - & - & - & 28 \\
Denormalized Table & - & - & - & - & - & 16 \\
Information Duplication & - & - & - & - & - & 1 \\
Redundant Column & - & - & - & - & - & 11 \\
No Domain Constraint & - & - & - & - & - & 0 \\
\hline Total: & 14764 & 63058 & 278 & 336 & - & 200
\end{tabular}

Table 3: Distribution of APs- Distribution of APs detected by SQLCHECK (S) and DBDEO (D) in queries collected from repositories on GitHub and written by the user study participants.

to the default behavior or lack of certain features in Django. Four teams are incorporating the fixes from SQLCHECK. Three teams are looking for alternate fixes. Three teams did not share their course of action. One team decided not to fix the reported APs given their application-specific requirements. Most of these teams were interested in understanding the implications of these APs and requested us to send patches. 


\begin{tabular}{rlrr}
\hline \# & GitHub Repo & \# AP Det & \# AP Rep \\
\hline 1 & Globaleaks & 10 & 2 \\
2 & Django-oscar & 12 & 2 \\
3 & Saleor & 10 & 2 \\
4 & Django-crm & 8 & 4 \\
5 & django-cms & 11 & 1 \\
\hline $\mathbf{1 7}$ & Total & $\mathbf{1 2 3}$ & $\mathbf{3 2}$
\end{tabular}

Table 4: Evaluation of sQLCHECK on Web Applications: The APs detected by sQLCHECK (\# APs Det) in a subset of 15 Django applications. We list the major APs that we reported (\# APs Rep).

\begin{tabular}{rlr}
\hline$\#$ & Kaggle Database & \# AP \\
\hline 1 & The History of Baseball & 41 \\
2 & Soccer Dataset & 20 \\
3 & Acad. Research from Indian Univ. & 17 \\
4 & Pesticide Data Program & 13 \\
5 & Board Games & 12 \\
\hline $\mathbf{3 1}$ & Total & $\mathbf{2 0 0}$ \\
\hline
\end{tabular}

Table 5: Evaluation of sQLCHECK on real-world databases: The APs detected by sQLCHECK in a subset of 31 Kaggle databases.

In one of these applications, SQLCHECK found APs that introduced by a third-party library. In another application, we found an existing issue related to the Too Many Joins AP. The developers found that replacing the ORM-generated query with a simpler, hand-written query greatly improved performance. This experiment illustrates the efficacy of SQLCHECK in assisting application developers in practice.

Data Analysis: We next evaluate the efficacy of sQlcheck in finding APs in real-world databases on Kaggle [26]. We download 31 SQLite databases and apply the data analysis rules of SQLCHECK on them (\$4.2). As shown in Table 5, sQLCHECK detects 200 APs across these databases (Ref. Section A). This experiment illustrates the efficacy of SQLCHECK in detecting APs by only analysing data (without queries).

\subsection{Limitations And Future Work}

Anti-Pattern Coverage, Discovery, and Evolution: SQLCHECK currently detects 26 types of APs. We intend to add support for more known APs in the future. However, it is unclear how to automatically discover new types of APs in SQL queries. Furthermore, the performance impact of an AP can evolve over time. For instance, the performance impact of the Adjacency list AP was prominent in PostgreSQL v9 $(5 \times)$. However, it is no longer significant $(1.1 \times)$ in v11.

Dialect Coverage and Query Repair: sQlcheck is designed to support multiple SQL dialects for higher utility. We accomplish this using a non-validating query parser (§4.1). However, it is infeasible to handle dialect-specific features, especially in complex queries. The usage of a nonvalidating query parser also restricts the set of queries wherein we can automatically rewrite the query to fix the AP. This is because we do not have enough syntactical information for query rewriting. We instead fall back on tailored textual fixes in these scenarios. We made this decision to increase the utility of SQLCHECK. The data analyzer ( $\$ 4.2)$ is built on top of SQLALCHEMY so that it can support diverse DBMSs (e.g., PostgreSQL, MySQL). Thus, the set of DBMSs that can be analyzed using ap-detect is constrained by those that are supported by SQLALCHEMY.

\section{Related Work}

Transforming Database Applications: Although program analysis has a long history in software engineering, it has not been extensively studied by the DBMS community. Recent research efforts have focused on transforming database-backed programs to improve performance $[9,19$, 45]. Ramachandra et al. present application transformations that enable asynchronous and batched query submission [37]. DBridge presents a set of holistic optimizations including query batching and binding, and automatic transformation of object-oriented code into synthesized queries [16, 37]. Cheung et al. describe techniques for batching queries to reduce the number of round trips between the application and database servers [7-10].

Object-Relational Mapping: Researchers have studied the impact of ORM on application design and performance [4-6, 43, 46]. Yang et al. perform a comprehensive study of performance issues in database applications using profiling techniques [47].

This paper is the first to explore the problems of automatically ranking and fixing APs in database applications.

\section{Conclusion}

In this paper, we presented SQLCHECK, a holistic toolchain for finding, ranking, and fixing APs in database applications. SQLCHECK leverages a novel AP detection algorithm that augments query analysis with data analysis. It improves upon DBDEO, the state-of-the-art tool for detecting AP, by using the overall context of the application to reduce false positives and negatives. SQLCHECK relies on a ranking model for characterizing the impact of detected APs. We discussed how SQLCHECK suggests fixes for high-impact AP using rule-based query refactoring techniques. Our empirical analysis shows that SQLCHECK enables developers to create more performant, maintainable, and accurate applications.

\section{Acknowledgements}

This work was supported in part by the U.S. National Science Foundation (IIS-1908984, IIS-1850342), Intel, and Alibaba. We thank Shamkant Navathe and our reviewers for their constructive feedback. We thank all of the contributors to sQLChEck: Venkata Kishore Patcha, Varsha Achar, Pooja Bhandary, Jennifer Ma, and Sri Vivek Vanga. 


\section{References}

[1] [n.d.]. Django. https://www.djangoproject.com.

[2] Andi Albrecht. 2019. sqlparse: A non-validating SQL parser module for Python . https://github.com/andialbrecht/sqlparse.

[3] Surajit Chaudhuri and Vivek Narasayya. 1998. AutoAdmin "what-if" index analysis utility. SIGMOD Record 27, 2 (1998), 367-378. https://doi.org/10.1145/276305.276337

[4] Tse-Hsun Chen. 2015. Improving the quality of large-scale database-centric software systems by analyzing database access code. In Proc. of ICDEW. IEEE, 245-249.

[5] Tse-Hsun Chen, Weiyi Shang, Zhen Ming Jiang, Ahmed E Hassan, Mohamed Nasser, and Parminder Flora. 2014. Detecting performance anti-patterns for applications developed using object-relational mapping. In Proc. of ICSE. ACM, 1001-1012.

[6] Tse-Hsun Chen, Weiyi Shang, Zhen Ming Jiang, Ahmed E Hassan, Mohamed Nasser, and Parminder Flora. 2016. Finding and evaluating the performance impact of redundant data access for applications that are developed using object-relational mapping frameworks. IEEE Transactions on Software Engineering 42, 12 (2016), 1148-1161.

[7] Alvin Cheung, Owen Arden, Samuel Madden, Armando Solar-Lezama, and Andrew C. Myers. 2013. StatusQuo: Making Familiar Abstractions Perform Using Program Analysis. In Proc. of CIDR.

[8] Alvin Cheung, Samuel Madden, and Armando Solar-Lezama. 2016. Sloth: Being lazy is a virtue (when issuing database queries). ACM Transactions on Database Systems (TODS) 41, 2 (2016), 8.

[9] Alvin Cheung, Samuel Madden, Armando Solar-Lezama, Owen Arden, and Andrew C Myers. 2014. Using Program Analysis to Improve Database Applications. IEEE Data Eng. Bull. 37, 1 (2014), 48-59.

[10] Alvin Cheung, Armando Solar-Lezama, and Samuel Madden. 2013. Optimizing database-backed applications with query synthesis. In SIGPLAN 48, 6 (2013), 3-14.

[11] Digital Equipment Corporation. 1992. SQL-92 Standard. https://www.contrib.andrew.cmu.edu/ shadow/sql/sql1992.txt.

[12] Ember Crooks. 2013. Why low cardinality indexes negatively impact performance.

https:/www.ibm.com/developerworks/data/library/techarticle/dm1309cardinal/index.html.

[13] Carlo Curino, Evan PC Jones, Raluca Ada Popa, Nirmesh Malviya, Eugene Wu, Sam Madden, Hari Balakrishnan, and Nickolai Zeldovich 2011. Relational cloud: A database-as-a-service for the cloud. (2011).

[14] Thomas H Davenport and DJ Patil. 2012. Data scientist. Harvard business review 90, 5 (2012), 70-76.

[15] Karl Dias, Mark Ramacher, Uri Shaft, Venkateshwaran Venkataramani, and Graham Wood. 2005. Automatic Performance Diagnosis and Tuning in Oracle. In $C I d R$.

[16] K Venkatesh Emani, Karthik Ramachandra, Subhro Bhattacharya, and S Sudarshan. 2016. Extracting equivalent sql from imperative code in database applications. In Proc. of SIGMOD. ACM, 1781-1796.

[17] Hermes Center for Transparency and Digital Human Rights. 2019. GlobaLeaks. https://www.globaleaks.org/.

[18] Goetz Graefe. 1993. Query evaluation techniques for large databases. In CSUR 25, 2 (1993), 73-169.

[19] Ravindra Guravannavar and S Sudarshan. 2008. Rewriting procedures for batched bindings. In Proceedings of VLDB 1, 1 (2008), 1107-1123.

[20] E Iee. 1990. Ieee standard glossary of software engineering terminology. (1990).

[21] Cunningham \& Cunningham Inc. 2014. C2 Wiki. http://wiki.c2.com/?AntiPatternsCatalog.

[22] Facebook Inc. 2019. ReactJS. https://reactjs.org.

[23] GitHub Inc. 2019. GitHub. https://github.com.
[24] Stitch Inc. 2018. The State of Data Science. https://www.stitchdata.com/resources/the-state-of-data-science/.

[25] Stack Exchange Inc. 2010. StackOverflow Wiki. https://stackoverflow.com/questions/346659/what-are-the-mostcommon-sql-anti-patterns.

[26] Kaggle. 2020. Kaggle. https://kaggle.com.

[27] Bill Karwin. 2010. SQL antipatterns: avoiding the pitfalls of database programming. Pragmatic Bookshelf.

[28] Sushil Kumar. 2003. Oracle Database 10g: The Self-Managing Database. White Paper.

[29] Eva Kwan, Sam Lightstone, Adam Storm, and Leanne Wu. 2002. Automatic Configuration for IBM DB2 Universal Database. Technical Report. IBM.

[30] David Lomet, Alan Fekete, Gerhard Weikum, and Mike Zwilling. 2009. Unbundling transaction services in the cloud. arXiv preprint arXiv:0909.1768 (2009)

[31] Dushyanth Narayanan, Eno Thereska, and Anastassia Ailamaki. 2005. Continuous Resource Monitoring for Self-predicting DBMS. In MASCOTS. 239-248.

[32] Pallets. 2019 . Python-Flask. http://flask.palletsprojects.com/en/1.1.x.

[33] PostgreSQL. 2014. Postgres Query Parsing. https://wiki.postgresql.org/wiki/Query_Parsing.

[34] PostgreSQL. 2019. Constraints in PostgreSQL. https:/www.postgresql.org/docs/current/ddl-constraints.html\#DDLCONSTRAINTS-CHECK-CONSTRAINTS.

[35] Python Software Foundation. 2019. Python. https://www.python.org.

[36] Python Software Foundation. 2019. PyPi. https://pypi.org.

[37] Karthik Ramachandra, Mahendra Chavan, Ravindra Guravannavar, and S Sudarshan. 2015. Program transformations for asynchronous and batched query submission. In TKDE 27, 2 (2015), 531-544.

[38] Raghu Ramakrishnan and Johannes Gehrke. 2003. Database Management Systems (3 ed.). McGraw-Hill, Inc., New York, NY, USA.

[39] Toby Segaran and Jeff Hammerbacher. 2009. Beautiful data: the stories behind elegant data solutions. " O’Reilly Media, Inc.".

[40] Microsoft SQL Server. 2019. Database Engine Tuning Advisor. https://docs.microsoft.com/en-us/sql/relationaldatabases/performance/database-engine-tuning-advisor?view=sqlserver-ver15.

[41] Tushar Sharma, Marios Fragkoulis, Stamatia Rizou, Magiel Bruntink, and Diomidis Spinellis. 2018. Smelly relations: measuring and understanding database schema quality. In Proc. of ICSE. ACM, 55-64.

[42] SQLAlchemy. 2019. SQLAlchemy. https://www.sqlalchemy.org/.

[43] Alexandre Torres, Renata Galante, Marcelo S Pimenta, and Alexandre Jonatan B Martins. 2017. Twenty years of object-relational mapping: A survey on patterns, solutions, and their implications on application design. Information and Software Technology 82 (2017), 1-18.

[44] Dana Van Aken, Andrew Pavlo, Geoffrey J Gordon, and Bohan Zhang. 2017. Automatic database management system tuning through large-scale machine learning. In SIGMOD. 1009-1024.

[45] Cong Yan and Alvin Cheung. 2016. Leveraging lock contention to improve OLTP application performance. In Proceedings of $V L D B$ 9, 5 (2016), 444-455

[46] Cong Yan, Alvin Cheung, Junwen Yang, and Shan Lu. 2017. Understanding Database Performance Inefficiencies in Real-world Web Applications. In Proc. of CIKM. ACM, 1299-1308.

[47] Junwen Yang, Cong Yan, Pranav Subramaniam, Shan Lu, and Alvin Cheung. 2018. How not to structure your database-backed web applications: a study of performance bugs in the wild. In Proc. of ICSE. IEEE, 800-810. 


\section{Appendix A Data Analysis}

In this experiment, we apply sQLCHECK's data analysis rules on 31 publicly-available SQLite databases from Kaggle. Table 6 lists the SQLite databases from Kaggle [26] that we use in this experiment along with the APs detected in these databases. We found 200 APs across 31 databases using SQLCHECK. The results of this experiment are discussed in $\S 8.4$.

\section{Appendix B Real World Applications}

We tested SQLCHECK on 15 actively developed, open-source applications based on the Django ORM [1]. In this experiment, we pass the SQL queries obtained from either running the application's integration tests or by manually interacting with the application to SQLCHECK. We rank the detected APs based on their impact and reported the ones with high impact. The APs detected are summarized in Table 7. The results of this experiment are discussed in §8.4.

\section{Appendix C Database Tuning Advisors}

There is a large body of work related to automated database administration tools geared towards optimizing: (1) database physical design, and (2) database knob configuration.
The former set of tools focus on selecting the best physical design (e.g., materialized views) for a given database that optimizes the target metrics (e.g., latency) and satisfies the budget constraints (e.g., hardware resources). These tools include Microsoft's AutoAdmin [3] and DETA [40] The latter set of tools instead focus on selecting the best knob configuration (e.g., buffer pool size) for a given DBMS. These tools include OtterTune [44] and vendor-specific offerings [15, 28, 29, 31].

Table 8 compares the core features of SQLCHECK and a tuning advisor (DETA). sQLCHECK complements DETA in that it focuses on other aspects of database applications besides physical design (e.g., logical design APs and query APs). We recommend the user to leverage the accurate feedback of the physical design tuning tool to fix the detected physical design APs, since it leverages the cost model of the query optimizer. SQLCHECK assists application developers in preventing APs during the application development phase by suggesting better alternatives (as opposed to tuning tools that are more often used post deployment). 


\begin{tabular}{|c|c|c|c|}
\hline \# & SQLite Database & \# AP & Detected Anti-Patterns \\
\hline 1 & Board Games & 12 & No Primary Key, Data in Metadata, Incorrect Datatype \\
\hline 2 & Pennsylvania Safe Schools Report & 1 & No Primary Key \\
\hline 3 & Soccer Dataset & 20 & Generic Primary Key, Data in Metadata, Missing Timezone, Multivalued Attribute \\
\hline 4 & SF Bay Area Bike Share & 11 & No \& Generic Primary Key, Incorrect Datatype, Missing Timezone, Denormalized Table \\
\hline 5 & US Baby Names & 2 & Generic Primary Key \\
\hline 6 & Pitchfork Music Data & 10 & No Primary Key, Missing Timezone, Information Duplication, Denormalized Table \\
\hline 7 & Acad. Research from Indian Univ. & 17 & No Primary Key, Incorrect Datatype, Redundant Column, Multivalued Attribute \\
\hline 8 & What.CD HipHop & 3 & No Primary Key, Multivalued Attribute \\
\hline 9 & Snap Meme-Tracker & 1 & Missing Timezone \\
\hline 10 & NIPS papers & 4 & Generic Primary Key, Denormalized Table \\
\hline 11 & US Wildfires & 2 & No Primary Key, Redundant Column \\
\hline 12 & Que from crossvalidated StackExc & 3 & No Primary Key \\
\hline 13 & The History of Baseball & 41 & No Primary Key, Data in Metadata, Incorrect Datatype, Multivalued Attribute \\
\hline 14 & Twitter US Airline Sentiment & 2 & Denormalized Table \\
\hline 15 & Hilary Clinton Emails & 8 & Generic Primary Key, Incorrect Datatype \\
\hline 16 & SEPTA - Regional Rail & 2 & Incorrect Datatype, Missing Timezone \\
\hline 17 & US Consumer finance Complaints & 9 & No Primary Key, Incorrect Datatype, Multivalued Attribute, Denormalized Table \\
\hline 18 & 1st GOP Debate Twitter Sentiment & 1 & Generic Primary Key \\
\hline 19 & SF Salaries & 2 & Generic Primary Key, Denormalized Table \\
\hline 20 & Freight Matrix Transportation & 5 & No Primary Key, Data in Metadata, Redundant Column \\
\hline 21 & WDIdata & 9 & No Primary Key, Multivalued Attribute \\
\hline 22 & Amazon Movie Reviews Dataset & 2 & No Primary Key, Multivalued Attribute \\
\hline 23 & UK Arms Export License & 3 & No Primary Key \\
\hline 24 & Amazon Fine Food Reviews & 1 & Generic Primary Key \\
\hline 25 & Stackoverflow Question Favourites & 1 & Multivalued Attribute \\
\hline 26 & Iron March $\sim$ & 1 & Redundant Column \\
\hline 27 & C\# Methods with Doc. Comments & 4 & Generic Primary Key \\
\hline 28 & Pesticide Data Program & 13 & No Primary Key, Incorrect Datatype, Redundant Column \\
\hline 29 & Monty Python Flying Circus & 4 & No Primary Key, Missing Timezone, Denormalized Table \\
\hline 30 & Twitter Conv. about Black Panther & 0 & - \\
\hline \multirow[t]{2}{*}{31} & 2016 US Election & 6 & No Primary Key, Data in Metadata, Denormalized Table \\
\hline & Total & 200 & \\
\hline
\end{tabular}

Table 6: Data Analysis Results A list of SQLite databases from Kaggle [26] with the APs detected by applying the data analysis rules in SQLCHECK.

\begin{tabular}{|c|c|c|c|c|c|c|c|}
\hline GitHub Repo & Stars & Contr. & Domain & \# AP & APs Reported & $\mathbf{R}$ & A \\
\hline Globaleaks & 741 & 22 & Whistleblower & 10 & 2 (No Foreign Key, Enumerated Types) & $\checkmark$ & $\checkmark$ \\
\hline Django-oscar & $4.1 \mathrm{k}$ & 217 & E-commerce & 12 & 2 (Rounding Errors, Index Overuse) & $\checkmark$ & $\checkmark$ \\
\hline Saleor & $6.5 \mathrm{k}$ & 139 & E-commerce & 10 & 2 (Multivalued Attribute, Index Overuse) & $\checkmark$ & $\checkmark$ \\
\hline Django-crm & 654 & 17 & CRM & 8 & 4 (Index Underuse, Index Overuse, Pattern Matching, No Domain Constraint) & $\checkmark$ & $\checkmark$ \\
\hline django-cms & $7.2 \mathrm{k}$ & 398 & CMS & 11 & 1 (Index Overuse) & $\checkmark$ & $\checkmark$ \\
\hline wagtail-autocomplete & 41 & 7 & Utility & 1 & 1 (Pattern Matching) & $\checkmark$ & $\checkmark$ \\
\hline shuup & $1.1 \mathrm{k}$ & 41 & E-commerce & 6 & 1 (Index Overuse) & $\checkmark$ & $\checkmark$ \\
\hline Pretix & 821 & 113 & E-commerce & 11 & 3 (Index Overuse, Pattern Matching, No Domain Constraint) & $\checkmark$ & $\checkmark$ \\
\hline Django-countries & 755 & 35 & Library & 1 & 1 (Multivalued Attribute) & $\checkmark$ & $\checkmark$ \\
\hline micro-finance & 55 & 8 & Finance & 8 & 4 (Index Underuse, Index Overuse, Pattern Matching, No Domain Constraint) & $\checkmark$ & $\checkmark$ \\
\hline bootcamp & $1.9 \mathrm{k}$ & 24 & Social Ntwrk & 5 & 1 (Index Overuse) & $\checkmark$ & $\checkmark$ \\
\hline NetBox & $6.2 \mathrm{k}$ & 118 & DCIM & 9 & 3 (Index Overuse, Pattern Matching, No Domain Constraint) & $\checkmark$ & $\checkmark$ \\
\hline Ralph & $1.3 \mathrm{k}$ & 43 & Asset Mgmt & 12 & 3 (Index Overuse, Pattern Matching, No Domain Constraint) & $\checkmark$ & $x$ \\
\hline Tiaga & $6.5 \mathrm{k}$ & 139 & E-commerce & 9 & 2 (Index Overuse, No Domain Constraint) & $\checkmark$ & $x$ \\
\hline wagtail & $8.4 \mathrm{k}$ & 397 & CMS & 10 & 2 (Index Overuse, No Domain Constraint) & $\checkmark$ & $x$ \\
\hline Total & & & & 123 & 32 & & \\
\hline
\end{tabular}

Table 7: Evaluation of sQLCHECK on real-world applications on GitHub: A list of open-source database applications on GitHub along with their popularity and domain, the APs (counts and names) detected by sQLCHECK, whether these APs have been reported (R) as GitHub Issues and acknowledged (A) by the contributors. 


\begin{tabular}{lcc}
\hline Supported Features & DETA & SQLCheck \\
\hline Index creation/destruction suggestions & $\checkmark$ & $\checkmark$ \\
Type of index to create based on workload & $\checkmark$ & $\times$ \\
Materialized view creation/destruction suggestions & $\checkmark$ & $\times$ \\
Suggestions tailored based on hardware constraints, workload, \& data distribution & $\checkmark$ & $\times$ \\
Table partitioning suggestions & $\checkmark$ & $\times$ \\
Column type suggestions based on data & $\times$ & $\checkmark$ \\
Query refactoring suggestions & $\times$ & $\checkmark$ \\
Alternate logical schema design suggestions & $\times$ & $\checkmark$ \\
Logical errors that may invalidate data integrity & $\times$ & $\checkmark$ \\
\hline
\end{tabular}

Table 8: SQLCheck v/s Microsoft DETA [40] Comparison of the core features of sQLCHECK against a physical design tuning advisor. 